\title{
A Simulation Study on Supply Chain Performance with Uncertainty Using Contract
}

\author{
Felix T. S. Chan, and H. K. Chan
}

\begin{abstract}
Traditional optimization techniques have been proposed in order to improve the performance of supply chains. Although these approaches have been shown to be effective, most of them were developed without considering uncertainties in order to simplify the analysis. In distributed supply chains, participating members are not under a sole control of a central authority, the problem is even more stringent due to incomplete information sharing. One way to improve the system performance is to achieve coordination among participating parties through establishment of contracts. The main objective of this paper is to propose a coordination mechanism with early order completion contract to minimize the negative impacts of demand uncertainty. Performance of the system is measured in terms of cost and fill rate. Simulation results indicate that the proposed contract approach is able to improve the performance measures of the system subjects to uncertain demand.
\end{abstract}

\section{INTRODUCTION}

Qupply chains consist of a network of participating $\mathcal{O}_{\text {organizations through upstream and downstream }}$ linkages, in different processes and activities that produce value in the form of products and services in the hands of the ultimate consumer [1]. Uncertainty takes the form of unforeseen demand variability, which in turn creates problems in planning, scheduling, and control of downstream entities [2]. Treating uncertainty is an important issue in supply chain modeling and analysis of the performance of the system [3]. Petrovic et al. [4] attempted to examine the effects of a serial supply chain in uncertain environment. Sources of uncertainty were customer demand and supplier reliability. The authors concluded that uncertain customer demand and uncertain supply delivery along the supply chain have great impact on supply chain behavior.

The main objective of this paper is to develop a coordination mechanism with early order completion contract, which aims to minimize the negative impacts of

Manuscript received October 28, 2004

F. T. S. Chan is with the Department of Industrial \& Manufacturing Systems Engineering, The University of Hong Kong, Pokfulam, Hong Kong (phone: +852 28597059; fax: +852 28586535; e-mail: ftschan@hkucc.hku.hk).

H. K. Chan is with Department of Industrial \& Manufacturing Systems Engineering, The University of Hong Kong, Pokfulam, Hong Kong (email: hkchan@ieee.org). the demand uncertainty. Simulation results by adopting the proposed algorithm will be presented. Performance of the system is measured in terms of cost and fill rate, which is referred to demand fill rate in this study. The organization of this paper is as follows: Section II reviews key literature and Section III presents the supply chain model along with its performance measures. Section IV summarizes the effects of demand uncertainty through a simulation study. This set of study is also treated as control experiments for the later simulation with early order completion contract. Section V presents the proposed coordination mechanism with early order completion contract. Section VI provides another set of simulation results to test the proposed contract approach subjects to demand uncertainty. Section VII is the concluding section.

\section{LITERATURE REVIEW}

Supply chain management relates to the coordination of products and information flows among various entities in the system [5]. Lee et al. [6] discussed and claimed that information that is transferred in the form of "order" tends to be distorted in supply chains and can misguide upstream members in both their inventory and production decisions. The magnitude of distortion tends to increases as one move upstream along a supply chain. They termed this phenomenon as the well-known "bullwhip effect". Consequently, additional inventory and production costs may be incurred. Due to their complex interactions, supply chain members can increase their own profits by coordinating with each others in order to fulfill the system goals [7].

Quantity / price discount is a common strategy to provide coordination channel among supply chain members [8 - 10]. Above literatures employed decentralized supply chain models in their studies. The research provided valuable insight in coordinating supply chain members in a distributed manner. One common weakness of the above research, however, is that deterministic demand is assumed and therefore, impact of system dynamics on the proposed model could not be studied. In fact, facing uncertain demand, retailers prefer to place an order late in most case [11] in order to gather enough time to collect more information, which is helpful to reduce demand forecast 
error. However, this results in insufficient production times and hence production cost (including penalty cost) would probably be increased.

Zhao et al. [12] proposed that early order commitments by retailers would benefit most in terms of cost reduction and service improvement of a supply chain. Although Zhao et al. [12] had demonstrated that early order commitments by retailers would be helpful to improve supply chain performances, it is in fact not easy to achieve under uncertain environment. On the other hand, once an order is established, the order can be completed earlier if both retailer and supplier are agreed to do so. "Early order completion" means a retailer can request the supplier to complete an inventory replenishment order at a defined quantity instead of waiting for the "full" quantity of the order. In other words, early order completion under contract may also be helpful to improve supply chain performance because back orders can be filled immediately. This can be achieved in the form of contract between the supplier and retailer. However, the effect of early order completion has not been addressed so far. In this connection, the paper sets out to investigate early order completion in the form of contract on supply chain performance, subject to demand uncertainty.

In fact, coordination can also exist in the form of contracting. However, little formal documentation exists describing how specific flexibility parameters have been arrived [13]. In fact, flexibility contract provides flexibility with no explicit penalty for exercise, by adopting constraints as a way to motivate appropriate behavior [13]. This philosophy is in line with solving Constraint Satisfaction Problem (CSP), which will be reviewed below.

Formally, Constraint Satisfaction Problem (CSP) is a triple [14]:

$$
\operatorname{CSP}(P)=(X, D, C)
$$

where $P=$ a constraint satisfaction problem;

$X=$ a set of variables such that $X=\left\{x_{1}, \ldots, x_{n}\right\}$;

$D=$ a set of domain such that $D=\left\{D_{l}, \ldots\right.$ $D_{n}$ \} and each variable $x_{i}$ of $X$ belongs to a finite set $D_{i}$, i.e. $x_{i} \in D_{i}$ for all $i$;

$C=$ a set of constraints restricting the values that the variables can simultaneously assign from $D$.

In other words, CSP is a problem with a set of variables, together with a set of possible values that can be assigned to each variable, and a list of constraints. The objective is to find values of the variables that satisfy every constraint. A feasible solution to a CSP is an assignment of a value from its domain to each variable, in such a way that every constraint is satisfied. Many problems, e.g. scheduling [15], in operational research falls within this general framework. There is no standard procedure to solve CSP but algorithms for solving CSPs can be broadly divided into two groups: problem reduction (or sometimes called consistency check), and search [13].

Distributed CSP (DCSP) is formally defined as a CSP in which variables and constraints are distributed among multiple automated agents [16]. Solving DCSP involves communication between agents by sending message and each agent has some variables and tries to determine their values so that the value assignment must satisfy inter-agent constraints, which are also distributed among agents [17]. Various application problems with distributed problem solving nature that are concerned with finding a consistent combination of agent actions can be formalized as DCSP [18]. In general, domain-specific coordination (or more specifically, negotiation) is necessary to balance the satisfaction among different agents [19].

In this paper, the philosophy of DCSP will be applied to solve the inventory management problem in distributed supply chains. In an inventory management problem, the variables of a retailer are the quantity and the time to reorder, where decision is constrained by its inventory position. For supplier, the variables are the quantity to produce and the time to ship (if the supplier has the flexibility), which is constrained by its own capacity and raw material supply. Domain of the variables is simply positive integers. Details of the coordination mechanism will be provided in Section $\mathrm{V}$.

\section{SIMULATION MODEL}

\section{A. The Supply Chain Model}

Recently, multi-agent modeling is widely recognized as an appropriate tooling to model distributed supply chain [20]. The distributed supply chain in this study is also modeled by multi-agent technique which was presented in Chan and Chan [20]. The supply chain under study consists of one retailer and four capacitated suppliers with single product type, which is a similar version that was used in Zhao et al. [5, 12]. Although the model is simple, it is good enough to analyses the effects of demand uncertainty and to demonstrate the usefulness of the proposed contract approach. Actual demand is generated every period. One period can be extended to one day, one week, or even one month so that demand can always be grouped in a per period basis. Therefore, only the demand quantity per period will be varied in this study and hence the period is fixed. As reported in Zhao et al. [5, 12], retailer makes an order to the suppliers based on the Economic Order Quantity (EOQ) model, which order quantity is given by equation (2) as follows:

$$
E O Q=\sqrt{\frac{2 k d}{h}}
$$


where $k=$ Ordering cost

$h=$ Inventory holding cost per unit per period

$d=$ Demand per period

In the EOQ model, demand is assumed as a constant value. This assumption is an ideal case that when no uncertainty exists in the system. By varying the demand per period, uncertainty of demand can be used as independent variables and the effects of the uncertainty can be quantified.

The simulation program is written in JAVA. Length of simulation is 465 periods while the first 100 periods are ignored for calculation in order to minimize the start-up effect. The final performance measures are based on the last 365 periods. If one period is equal to one day, then the effective length of simulation run is one year. Each simulation setting will be run with 10 different random seeds and the average is reported in order to minimize the random effect.

\section{B. Cost Structure}

Total cost of the system is calculated for each simulation run. The model and cost structure of this study is similar to the one presented by Zhao et al. $[5,12]$. In fact, the effects of demand uncertainty that will be presented in the subsequent sections are in line with the findings of their studies. Therefore, the model in this study is supported indirectly. The total cost consists of components stated as follows:

- Product cost: it is charged per unit basis;

- Ordering cost: it is charged to the retailer whenever an order is placed by the retailer;

- Transportation cost: it is charged to the retailer whenever an order is sent to the retailer. In other words, it can be treated as another part of the ordering cost;

- Setup cost: it is charged to the supplier whenever an order is confirmed;

- Inventory holding cost: it is calculated per unit per day; and

- Back order cost: it is calculated whenever inventory is not enough to cover demand and is calculated per unit per day.

\section{Demand Generation}

As mentioned in Section III.A, demand is generated every period. The demand is calculated base on the following equation that is similar to the one used in Zhao et al. $[5,12]$ :

$$
\begin{aligned}
\text { Demand }= & \text { Base }+ \text { Noise } x \text { Normal } \\
= & \text { Base }+(\text { Demand Error }+ \text { Demand Variation } x \\
& \text { Snormal })
\end{aligned}
$$

where Snormal is a random variable of standard normal.

In this study, demand uncertainty is modeled as a normal distribution. The mean and standard deviation of the function is equal to the demand error and demand variation in equation (3) respectively. The two parameters will be varied, i.e. they are the independent variables in this study. Demand error is assumed to be varied from $-30 \%$ of the base value to $+30 \%$ of the base value, at $10 \%$ interval (i.e. 7 levels). Demand variation will be varied from $0 \%$ of the based value to $30 \%$ of the base value, also at $10 \%$ interval (i.e. 4 levels). In fact, more levels of uncertainties have been varied and tested but the results are consistent with different uncertainty levels. Therefore, simulation results against those levels mentioned above will be presented.

\section{Performance Measures}

In order to analyze the effects of demand uncertainty and the usefulness of the proposed heuristic system, total system cost and fill rate of the whole system are recorded as performance measures (i.e. dependent variables).

\section{EFFECTS OF DEMAND UNCERTAINTY}

As mentioned in Section III.C, there are two independent variables - demand error and demand variation. The former is modeled as 7 levels and the latter is modeled as 4 levels. Therefore, there are $7 \times 4=28$ combinations of each simulation run. Important results are summarized below.

\section{A. Effects of Demand Uncertainty on Total System Costs}

Fig. 1 depicts the simulation results of percentage increase in total system cost against demand error of the system.

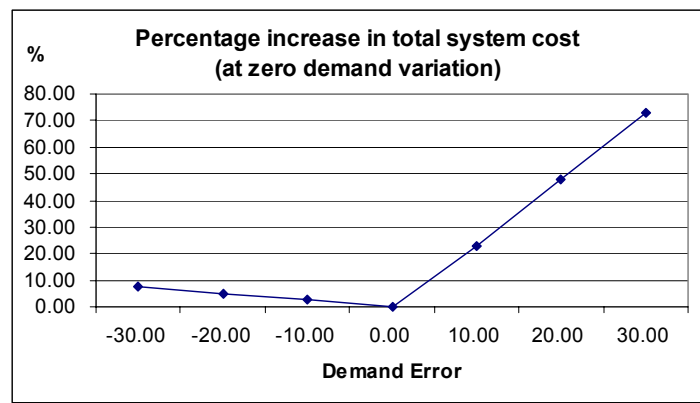

(a). Demand variation $=0 \%$

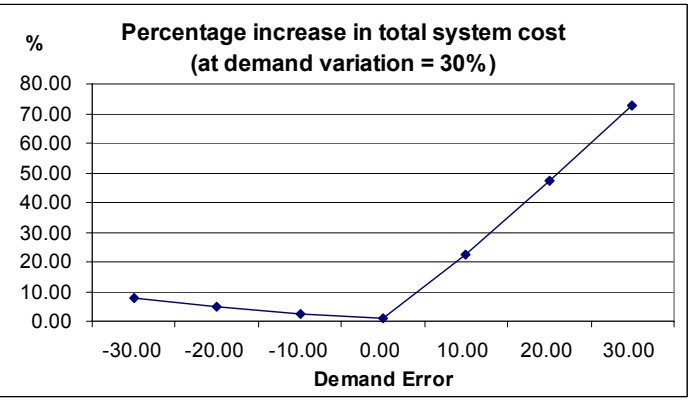

(b). Demand variation $=30 \%$

Fig. 1. Impact of demand error on total system cost 
Please be reminded that the percentage increase in total system cost is reference to zero demand error and zero demand variation, which is an ideal situation without any uncertainty. Although only the results when demand variations equal to zero (Fig. 1a) and 30\% (Fig. 1b) are included, the trend is similar for different values of demand variation (i.e. $10 \%$ and $20 \%$ ) so that they are omitted for simplicity.

It may be observed that the shape of Fig. 1(a) and Fig. 1(b) are the same. It is due to the fact that the total cost increased is more sensitive to the demand error, than to the demand variation (results not shown here).

Obviously, the total system cost is increasing with the absolute value of the demand error. From both Fig. 1(a) and (b), the effect is more significant on the right hand side (i.e. positive demand error) than on the left hand side (i.e. negative demand error). Back order costs are the main contribution to the system cost increased on the right hand side, whereas inventory costs account for the system cost increased on the left hand side. In a long run, increasing suppliers' capacity, or equivalently, introducing more suppliers can reduce the effect of the system cost increased due to positive demand error. However, the additional cost due to this shift should be kept at minimal in a short run by some remedy actions.

\section{B. Effects of Demand Uncertainty on Fill Rate}

Fig. 2 shows the fill rate of the system against different demand variation at zero demand error. The reason to show only the case of zero demand error is because fill rates of other demand errors have been saturated. This is because there is a larger deviation between the mean capacity of the system and the actual mean capacity. In other words, once back orders accumulate to a certain level, the system is unable to recover the back order any more. Therefore, only the results at zero demand error is shown. From Fig. 2, the fill rate is decreasing as the demand variation is increasing, as expected. Decrease in fill rate may result in poor customer satisfaction.

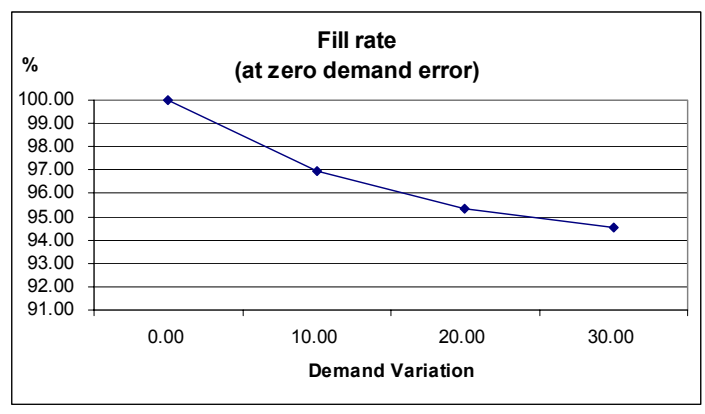

Fig. 2. Impact of demand variation on fill rate of the system (at zero demand error)

\section{THE COORDINATION MECHANISM}

From the results presented in Section IV, if the system demand of a supply chain is uncertain, the system is suffering from additional cost, and deterioration in fill rate. Particularly, when the demand error is positive, the inventory level would not be able to cover the required demand. Consequently, a large back order would be accumulated, which in turn induces a higher cost. In this connection, it is wise to implement a dynamic inventory replenishment policy to reduce the back order. In this study, a coordination mechanism is developed to reduce the impact of demand uncertainty by lowering the back order quantity, hence cost, and to improve the fill rate at zero demand error. The philosophy behind the mechanism is the principle of DCSP as described in Section II. The procedures are highlighted as follows:

1. Instead of requesting a fix EOQ from the retailer, a contract is made between the retailer and supplier with an additional parameter, which is called early order completion quantity (EOCQ). This is an important factor that will be examined later. The contract itself is a DCSP. The objective is to determine when to complete the order and how many (EOQ or EOCQ) to be shipped subject to the inventory constraints. As long as the retailer requests the supplier to complete the order, and if the supplier has enough inventories to fulfill the request (i.e. inventory larger than EOCQ), the supplier will ship that quantity and the contract is ended, i.e. the DCSP is solved.

2. When a period is elapsed, the retailer calculates a variable called "inventory tightness" to reflect the uncertain situation, using equation (2). The variable is monitored continuously at the beginning of each period.

$$
\text { Inventory Tightness }=\frac{\text { Current Inventory }}{\text { Mean demand } x d}
$$

where $d$ is the number of days to receive the next order as scheduled.

3. If this variable is higher than 1 , it is very likely that the inventory is enough to cover the actual demand before arrival of next order. No action will be enforced.

4. On the other hand, if the variable is less than 1, the retailer will seek a supplier with the highest inventory on hand. The retailer will send a message to the supplier to request for early order completion.

5. If the inventory level of the supplier is less than the EOCQ, the supplier will send a reject message (i.e. the DCSP cannot be solved at this particular moment) to the retailer and then no action will be imposed.

6. On the other hand, if the inventory level of that supplier is high than the EOCQ, the supplier will send a accept message to the retailer and then that quantity of inventory will be shipped to the retailer (i.e. the DCSP can be solved). Any costs that are incurred in 
this transaction are charged to the retailer.

7. If there is no need to complete the order earlier, the order will be completed according to the traditional EOQ model.

If the above procedures map to the DCSP as described in Section II, the DCSP is modeled as follows:

$$
\operatorname{DCSP}(P)=(X, D, C)
$$

where $P=$ the inventory replenishment problem; $X=$ a set of variables such that $X=\left\{x_{1}, x_{2}\right.$, $\left.x_{3}\right\}$, where $x_{1}$ is the variable inventory tightness, $x_{2}$ is the quantity of the contract to be completed, and $x_{3}$ is the inventory of the supplier;

$D=$ a set of domain such that $D=\left\{D_{1}, D_{2}\right.$, $\left.D_{3}\right\}$, where $D_{1}$ is set of a real number, $D_{2}$ is a set of positive integers, and $D_{3}$ is a finite set consists of 0 , EOCQ and EOQ;

$C=\mathrm{a}$ set of constraints but the constraint can be transformed as a single constraint $C_{1}:\left\{\left(x_{1}>=1\right.\right.$ and $\left.x_{2}=0\right)$ or (EOQ $>x_{3}$ $>=$ EOCQ and $x_{2}=$ EOCQ) or $(\mathrm{EOQ}<=$ $x_{3}$ and $\left.\left.x_{2}=\mathrm{EOQ}\right)\right\}$.

A simulation study was carried out to test the proposed algorithm. Details will be discussed in Section VI.

\section{SIMULATION RESULTS AND DISCUSSIONS}

Since the coordination mechanism, as discussed in Section V, pinpoints the impacts of positive demand error, the procedure will not be triggered if demand error is negative. Therefore, only the data obtained by varying positive demand error will be presented. As mentioned above, the EOCQ is a crucial parameter that will be varied. In fact, it is set as a percentage of the quantity from EOQ model in this study. Different settings have been tested as sensitivity analysis and the results in percentage improvement of the performance measures will be presented.

\section{A. Total Unit Cost}

Table 1 shows the percentage improvement by using the coordination mechanism. Negative value means improvement (i.e. cost decrease), while positive value means cost is increased. Improved values are highlighted (bold and italic) as well to improve readability. Unit cost is used because the total output quantity is different after the heuristic rule is triggered. Therefore, it is only fair to compare different situations by the unit cost.

Results indicate that if the EOCQ value is sufficiently large (greater than $20 \%$ of EOQ values in this study), unit cost would be decreased when demand error is non-zero. This is because whenever the rule is triggered, part of the inventory from suppliers will be shipped to the retailers to recover any back order immediately. This action leads to reduction in supplier inventory and retailer back order, and hence total system cost. When the EOCQ is set at $60 \%$ of the EOQ quantity, the improvement is the best among the settings under evaluation. In fact, this is related to a dilemma that when the rule is triggered, backorder cost can be reduced while additional management costs are incurred. If the EOCQ value is too low, additional management cost cannot be compensated by the reduction in back order cost.

TABLE 1.

Percentage Improvement in Total Unit Cost

\begin{tabular}{|c|c|c|c|c|}
\cline { 2 - 5 } \multicolumn{1}{c|}{} & \multicolumn{4}{c|}{ Demand Variation } \\
\hline $\begin{array}{c}\text { Demand } \\
\text { Error }\end{array}$ & $0 \%$ & $10 \%$ & $20 \%$ & $30 \%$ \\
\hline $0 \%$ & 0.00 & 1.81 & 2.16 & 2.22 \\
\hline $10 \%$ & 4.62 & 4.62 & 4.63 & 4.63 \\
\hline $20 \%$ & 3.47 & 3.48 & 3.49 & 3.49 \\
\hline $30 \%$ & 2.66 & 2.67 & 2.67 & 2.67 \\
\hline
\end{tabular}

(a) EOCQ value $=20 \%$ of EOQ

\begin{tabular}{|c|c|c|c|c|}
\cline { 2 - 5 } \multicolumn{1}{c|}{} & \multicolumn{4}{|c|}{ Demand Variation } \\
\hline $\begin{array}{c}\text { Demand } \\
\text { Error }\end{array}$ & $0 \%$ & $10 \%$ & $20 \%$ & $30 \%$ \\
\hline $0 \%$ & 0.00 & 1.14 & 1.07 & 0.90 \\
\hline $10 \%$ & -1.19 & -1.17 & -1.14 & -1.10 \\
\hline $20 \%$ & -1.35 & -1.35 & -1.35 & -1.35 \\
\hline $30 \%$ & -1.46 & -1.46 & -1.46 & -1.46 \\
\hline
\end{tabular}

(b) EOCQ value $=40 \%$ of EOQ

\begin{tabular}{|c|c|c|c|c|}
\cline { 2 - 5 } \multicolumn{1}{c|}{} & \multicolumn{4}{c|}{ Demand Variation } \\
\hline $\begin{array}{c}\text { Demand } \\
\text { Error }\end{array}$ & $0 \%$ & $10 \%$ & $20 \%$ & $30 \%$ \\
\hline $0 \%$ & 0.00 & 1.12 & 0.88 & 0.66 \\
\hline $10 \%$ & $\mathbf{- 0 . 8 5}$ & $\mathbf{- 0 . 8 5}$ & $\mathbf{- 0 . 8 4}$ & $\mathbf{- 0 . 8 2}$ \\
\hline $20 \%$ & $\mathbf{- 0 . 9 4}$ & $\mathbf{- 0 . 9 4}$ & $\mathbf{- 0 . 9 4}$ & $\mathbf{- 0 . 9 4}$ \\
\hline $30 \%$ & $\mathbf{- 1 . 0 0}$ & $\mathbf{- 1 . 0 0}$ & $\mathbf{- 1 . 0 0}$ & $\mathbf{- 1 . 0 0}$ \\
\hline
\end{tabular}

(c) EOCQ value $=50 \%$ of EOQ

\begin{tabular}{|c|c|c|c|c|}
\cline { 2 - 5 } \multicolumn{1}{c|}{} & \multicolumn{4}{c|}{ Demand Variation } \\
\hline $\begin{array}{c}\text { Demand } \\
\text { Error }\end{array}$ & $0 \%$ & $10 \%$ & $20 \%$ & $30 \%$ \\
\hline $0 \%$ & 0.00 & 1.11 & 0.78 & 0.48 \\
\hline $10 \%$ & $\mathbf{- 2 . 0 7}$ & $\mathbf{- 2 . 0 7}$ & $\mathbf{- 2 . 0 5}$ & $\mathbf{- 2 . 0 2}$ \\
\hline $20 \%$ & $\mathbf{- 1 . 9 9}$ & $\mathbf{- 1 . 9 9}$ & $\mathbf{- 1 . 9 9}$ & $\mathbf{- 1 . 9 9}$ \\
\hline $30 \%$ & $\mathbf{- 1 . 9 4}$ & $\mathbf{- 1 . 9 4}$ & $\mathbf{- 1 . 9 4}$ & $\mathbf{- 1 . 9 4}$ \\
\hline
\end{tabular}

(d) $\mathrm{EOCQ}$ value $=60 \%$ of EOQ

\begin{tabular}{|c|c|c|c|c|}
\hline \multicolumn{1}{c|}{} & \multicolumn{4}{|c|}{ Demand Variation } \\
\hline $\begin{array}{c}\text { Demand } \\
\text { Error }\end{array}$ & $0 \%$ & $10 \%$ & $20 \%$ & $30 \%$ \\
\hline $0 \%$ & 0.00 & 1.10 & 0.83 & 0.63 \\
\hline $10 \%$ & $\mathbf{- 0 . 6 6}$ & $\mathbf{- 0 . 6 6}$ & $\mathbf{- 0 . 6 6}$ & $\mathbf{- 0 . 6 4}$ \\
\hline $20 \%$ & $\mathbf{- 0 . 7 4}$ & $\mathbf{- 0 . 7 3}$ & $\mathbf{- 0 . 7 3}$ & $\mathbf{- 0 . 7 3}$ \\
\hline $30 \%$ & $\mathbf{- 0 . 7 9}$ & $\mathbf{- 0 . 7 9}$ & $\mathbf{- 0 . 7 9}$ & $\mathbf{- 0 . 7 8}$ \\
\hline
\end{tabular}

(e) EOCQ value $=80 \%$ of EOQ 


\section{B. Fill Rate}

The results of the fill rate are quite different. As mentioned before, when demand error is too large, the system is unable to recover the back order quantity. Therefore, no improvement can be found when demand error is more than $10 \%$. However, the proposed algorithm would not worsen the performance of the system in terms of fill rate. Nevertheless, attention should be paid on zero demand error. Improvement can be found at any EOCQ values when the demand error is zero. That means at zero demand error, the proposed algorithm succeeded in improving the fill rate. Fig. 3 illustrates the fill rate at different EOCQ values. The values of the legend are the EOCQ value in percentage of the EOQ quantity.

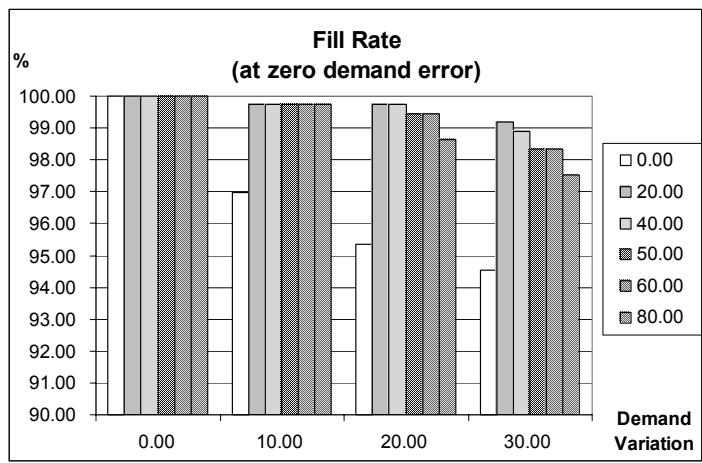

Fig. 3. Impact of demand variation on fill rate of the system with different EOCQ values (at zero demand error)

\section{CONCLUSION}

A coordination mechanism with early order completion contract is proposed in this paper to react with demand uncertainty in a distributed supply chain. Simulation results show that different performance measures can be improved against different EOCQ value of the proposed algorithm. However, as a rule of thumb, the EOCQ value can be set as half of the EOQ quantity, i.e. $40 \%$ to $60 \%$, in order to gain the maximum benefit when demand error is non zero. When demand error is zero, a dilemma has been encountered to improve the fill rate by adopting the algorithm or do nothing to maintain a relatively low costs impact due to demand variation.

One of the limitations of this research is that participating companies in the supply chain under study are willing to be coordinated according to the proposed algorithm. In fact, the algorithm only enforces some managerial actions and hence this assumption is easy to achieve.

\section{REFERENCES}

[1] M. Christopher, Logistics and Supply Chain Management. London: Pitman, 1998.
[2] M. Fisher, J. Hammond, W. Obermeyer, and A. Raman, "Configuring a supply chain to reduce the cost of demand uncertainty," Production and Operations Management, vol. 6, no. 3, pp. 211-225, 1997.

[3] D. Petrovic, "Simulation of supply chain behaviour and performance in an uncertain environment," International Journal of Production Economics, vol. 71, no. 1-3, pp. 429-438, 2001.

[4] D. Petrovic, R. Roy, and R. Petrovic, "Supply chain modelling using fuzzy sets," International Journal of Production Economics, vol. 59, no. 1-3, pp. 443-453, 1999

[5] X. Zhao, J. Xie, and J. Leung, "The impact of forecasting model selection on the value of information sharing in a supply chain," European Journal of Operational Research, vol. 142, no. 2, pp. 321344, 2002.

[6] H. L. Lee, V. Padmanabhan, and S. Whang, "Information Distortion in a Supply Chain: The Bullwhip Effect," Management Science, vol. 43, no. 4, pp. 546-558, 1997.

[7] Z. K., Weng, and T. McClurg, "Coordinated ordering decisions for short life cycle products with uncertainty in delivery time and demand," European Journal of Operational Research, vol. 151, no. 1, pp. 12-24, 2003.

[8] C. L. Munson, and M. J. Rosenblatt, "Coordinating a three-level supply chain with quantity discounts," IIE Transactions, vol. 33, no. 5, pp. 371-384, 2001.

[9] S. Viswanathan, and R. Piplani, "Coordinating supply chain inventories through common replenishment epochs," European Journal of Operational Research, vol. 129, no. 2, pp. 277-286, 2001.

[10] T. D. Klastorin, K. Moinzaheh, and J. Son, "Coordinating orders in supply chains through price discounts," IIE Transactions, vol. 34, no. 8, pp. 679-689, 2002

[11] J. Chen, and L. Xu, "Coordination of the Supply Chain of Seasonal Products," IEEE Transactions on Systems Man, and Cybernetics Part A, vol. 31, no. 6, pp. 524-531, 2001.

[12] X. Zhao, J. Xie, and R. S. M. Lau, "Improving the supply chain performance: use of forecasting models versus early order commitments," International Journal of Production Research, vol. 39, no. 17, pp. 3923-3939, 2001

[13] A. A. Tsay, "The Quantity Flexibility Contract and SupplierCustomer Incentives," Management Sciences, vol. 45, no. 10, pp. 1339-1358, 1999.

[14] S. C. Brailsford, C. N. Potts, and B. M. Smith, "Constraint satisfaction problems: Algorithms and applications," European Journal of Operational Research, vol. 119, no. 3, pp. 557-581, 1999.

[15] W. P. M. Nuijten, and E. H. L. Aarts, "A computational study of constraint satisfaction for multiple capacitated job shop scheduling," European Journal of Operational Research, vol. 90, no. 2, pp. 269284, 1996.

[16] M. Yokoo, E. H. Durfee, T. Ishida, and K. Kuwabara, "The Distributed Constraint Satisfaction Problems: Formalization and Algorithms," IEEE Transactions on Knowledge and Data Engineering, vol. 10, no. 5, pp. 673-685, 1998.

[17] M. Yokoo, and K. Hirayama, "Algorithms for Distributed Constraint Satisfaction: A Review," Autonomous Agents and Multi-Agent Systems, vol .3, no. 2, pp. 185-207, 2000.

[18] M. Yokoo, and K. Hirayama, "Distributed Constraint Satisfaction Algorithm for Complex Local Problems," in Proceedings of the 3rd International Conference of Multi-Agent Systems, La Villette, Paris, France, pp. 372-379, July 3-7, 1998.

[19] H. Wang, S. Liao, and L. Liao, "Modeling constraint-based negotiating agents," Decision Support Systems, vol. 33, no. 2, pp. 201-217, 2002.

[20] F. T. S. Chan, and H. K. Chan, "A New Model for Manufacturing Supply Chain Networks: a Multiagent Approach," Proceedings of the Institution of Mechanical Engineers Part B: Journal of Engineering Manufacture, vol. 218, no. 4, pp. 443-454, 2004. 